\title{
Mapping of axial strain in InAs/InSb heterostructured nanowires
}

Cite as: Appl. Phys. Lett. 107, 093103 (2015); https://doi.org/10.1063/1.4929979

Submitted: 01 June 2015 . Accepted: 18 August 2015 . Published Online: 01 September 2015

Atanu Patra, Jaya Kumar Panda, Anushree Roy, Mauro Gemmi (D), Jérémy David (iD), Daniele Ercolani, and Lucia Sorba
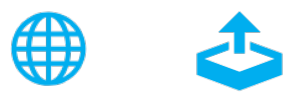

\section{ARTICLES YOU MAY BE INTERESTED IN}

Manipulation of polarization anisotropy in bare InAs and InAs/GaSb core-shell nanowires Applied Physics Letters 112, 153104 (2018); https://doi.org/10.1063/1.5021714

Spin-phonon coupling in scandium doped gallium ferrite

Journal of Applied Physics 117, 123915 (2015); https://doi.org/10.1063/1.4916602

Band parameters for III-V compound semiconductors and their alloys

Journal of Applied Physics 89, 5815 (2001); https://doi.org/10.1063/1.1368156

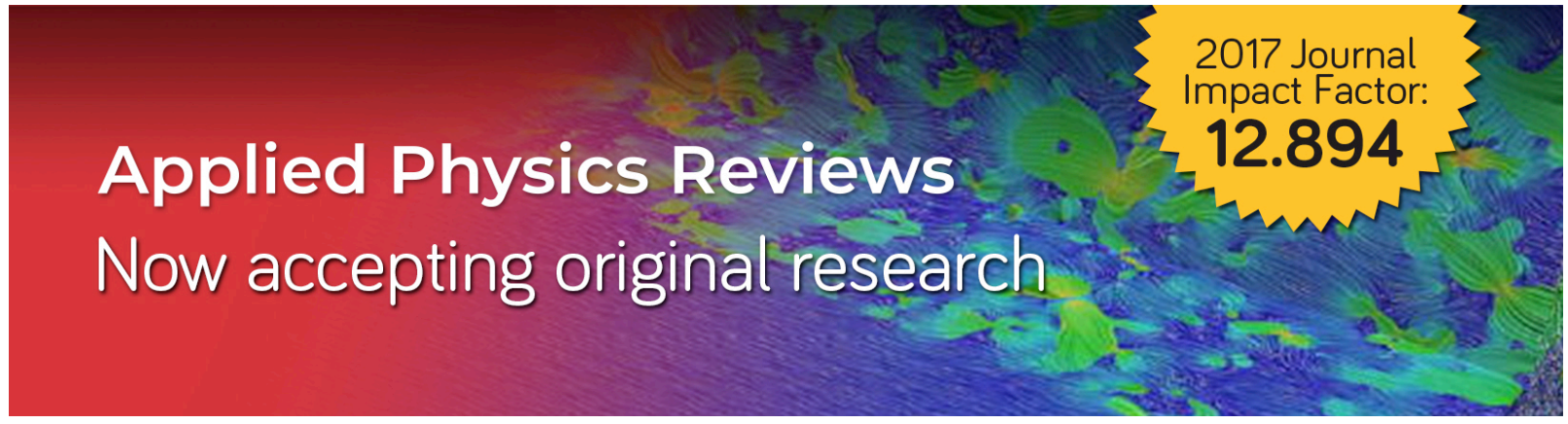




\title{
Mapping of axial strain in InAs/lnSb heterostructured nanowires
}

\author{
Atanu Patra, ${ }^{1}$ Jaya Kumar Panda, ${ }^{1}$ Anushree Roy, ${ }^{1, a)}$ Mauro Gemmi, ${ }^{2}$ Jérémy David, ${ }^{2}$ \\ Daniele Ercolani, ${ }^{3}$ and Lucia Sorba ${ }^{3, b)}$ \\ ${ }^{1}$ Department of Physics, Indian Institute of Technology Kharagpur, Kharagpur 721 302, India \\ ${ }^{2}$ Center for Nanotechnology Innovation@ NEST, Istituto Italiano di Tecnologia, Piazza S. Silvestro 12, \\ I-56127 Pisa, Italy \\ ${ }^{3}$ NEST-Istituto Nanoscienze-CNR and Scuola Normale Superiore, Piazza S. Silvestro 12, I-56127 Pisa, Italy
}

(Received 1 June 2015; accepted 18 August 2015; published online 1 September 2015)

\begin{abstract}
The article presents a mapping of the residual strain along the axis of InAs/InSb heterostructured nanowires. Using confocal Raman measurements, we observe a gradual shift in the transverse optical phonon mode along the axis of these nanowires. We attribute the observed shift to a residual strain arising from the $\mathrm{InAs} / \mathrm{InSb}$ lattice mismatch. We find that the strain is maximum at the interface and then monotonically relaxes towards the tip of the nanowires. We also analyze the crystal structure of the InSb segment through selected area electron diffraction measurements and electron diffraction tomography on individual nanowires. (C) 2015 AIP Publishing LLC.
\end{abstract}

[http://dx.doi.org/10.1063/1.4929979]

Recent times semiconductor research and development sectors embraced the use of III-V semiconductor nanowires (NWs) for high speed devices, ${ }^{1}$ high frequency electronics, ${ }^{2}$ and spin related applications. ${ }^{3}$ Among these, InSb NWs draw a special attention. From the basic physics point of view, finding Majorana fermions in InSb NWs in the presence of s-wave superconductors ${ }^{4}$ or the manipulation of strong spinorbit coupled qubit states ${ }^{5}$ have led to new dimensions in the research on this system.

The main hindrance in using lattice mismatched semiconductor nanostructures in any application is the occurrence of strain-induced defects in the system. However, it is claimed that the interfacial strain, which mostly appears due to the lattice mismatch of the NW materials with the available substrates, relaxes within few nanometers across the interface. ${ }^{6-9}$ For example, in a recent report ${ }^{6}$ using scanning transmission electron microscopy along with computer simulation and geometric phase analysis, it has been demonstrated that in InAs/InSb NWs the interfacial strain relaxes within $10 \mathrm{~nm}$ via both elastic deformation (misfit dislocations) and plastic deformation (plane bending). The misfit dislocations are observed at the center of the NWs, whereas the interfacial strain relaxes via the plane bending near the edge of the NWs.

In above references, electron diffraction measurements have been used to quantify the strain in NWs. Though, it is a direct probe to estimate strain by measuring lattice distortion, the resolution of the micrographs limits the measurable strain in a crystal structure. In general, using this technique, one is able to measure the strain of more than $1 \%$ in the crystal structure of semiconductor NWs. ${ }^{8,10}$ In comparison to electron diffraction measurements, Raman spectroscopy is more sensitive to the local strain in the crystal structure. There are quite a few reports in the literature in which the strain in the crystal structure of III-V semiconductor NWs has been demonstrated using Raman scattering measurements. ${ }^{11-13}$ However, it is to

\footnotetext{
${ }^{\text {a)} E m a i l: ~ a n u s h r e e @ p h y . i i t k g p . e r n e t . i n ~}$

b)Email: lucia.sorba@nano.cnr.it
}

be kept in mind that it is non-trivial to map the strain along the axis of the NWs, as the far field diffraction of light limits the spatial resolution of Raman scattering measurements. In this article, we employ confocal Raman spectroscopy to measure the strain in the crystal structure, along the axis of the InAs/InSb NWs, grown by chemical beam epitaxy (CBE) technique. The selected area electron diffraction (SAED) measurements and electron diffraction tomography (EDT) are exploited to measure the extent of lattice distortion in individual $\mathrm{NW}$.

$\mathrm{InAs} / \mathrm{InSb} \mathrm{NW}$ growths were performed by Au-assisted CBE technique. The details of the growth procedure are available as Sec. S1 in the supplementary material. ${ }^{14}$ Figure 1 shows a $45^{\circ}$ tilted scanning electron micrograph of vertically aligned [111] InAs/InSb NWs. As can be observed, NWs are composed of two sections: the lower smaller-diameter one is the InAs stem, while the upper one is the InSb segment and shows a diameter comparable with that of the catalyst. For more details refer to Sec. S2 in the supplementary material. ${ }^{14}$ The investigated InSb segments have zincblende crystal structure, a length of $\sim 4.0 \pm 0.2 \mu \mathrm{m}$ and a diameter $170 \pm 30 \mathrm{~nm}$. A detail morphological and structural analysis of the heterostructured InAs/InSb NWs are reported in the previous paper ${ }^{8}$ by some of the authors of this article.

Confocal Raman measurements were carried out in back-scattering geometry. In a confocal microscope, a pinhole is used in the optically conjugate plane in front of the detector to eliminate the out-of-focus light from the sample. Thus, it helps in improving the spatial resolution of the depth profiling by Raman scattering measurements. A pin-hole with a diameter of $50 \mu \mathrm{m}$ has been used. We worked with a confocal microscope (model BX41, Olympus, Japan) and $100 \times$ objective lens (numerical aperture 0.9) for focusing the laser beam on standing NWs. For this objective lens, the laser spot size is $1 \mu \mathrm{m}$ (model specification) at the focal plane. All Raman scattering results are from the ensemble average of 5-6 NWs. The detail of the spectrometer is available as Sec. S3 in the supplementary material. ${ }^{14}$ 


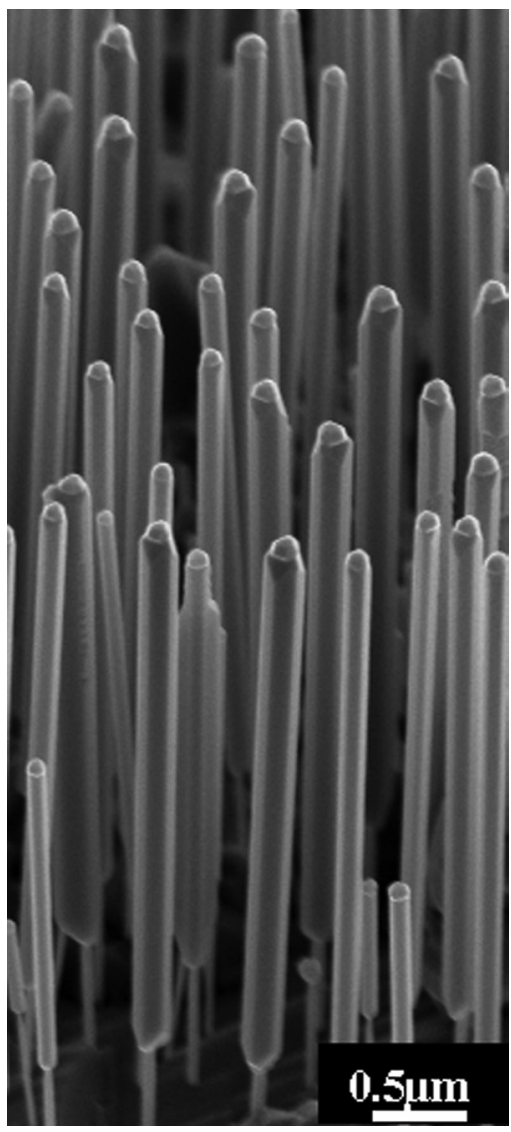

FIG. 1. $45^{\circ}$ tilted SEM image of aligned InAs/InSb NWs.

Because of far field diffraction limit of light, even in confocal Raman spectroscopy it is necessary to calibrate the depth resolution of the measurements. We used bulk InSb to estimate the spatial resolution of our confocal Raman measurements. We measured the ratio of intensity of Raman scattered light through the pinhole $\left(\mathrm{I}_{\mathrm{CF}}\right)$ and without the pinhole $\left(\mathrm{I}_{0}\right)$, at different distances $\mathrm{z}$ from the focal plane of the objective lens. The FWHM of the plot of this ratio $v s \mathrm{z}$ provides the measure of the depth resolution for the given pinhole. ${ }^{15}$ Figure 2 presents the measured step response curve for our experimental setup. From this calibration we obtain a depth resolution of $1.2 \mu \mathrm{m}$.

Keeping the same experimental conditions, employed for the calibration, we investigated vertically aligned InAs/ InSb NWs (shown in Figure 1). The laser light was first focused at the bottom of the InSb segment of the standing NWs (i.e., in the portion of the InSb segment near the interface with the InAs stem). The sample stage was then moved down manually by steps of $0.5 \mu \mathrm{m}$ [using the fine adjustment of the focusing micrometer screw of the microscope] to access different points along the axis of the InSb segments, from the InSb base towards the tip. Refer to the schematic diagram shown in the inset of Figure 2.

We performed the SAED measurements on a Zeiss Libra 120 operating at $120 \mathrm{kV}$. The measurements have been performed with the Digital Micrograph software by Gatan, using intensity profiles along the [111] and [1-11] directions, both having equivalent cubic symmetry.

The evolution of Raman spectra along the length of the NWs is shown in Figure 3(a). The bottom-most spectrum

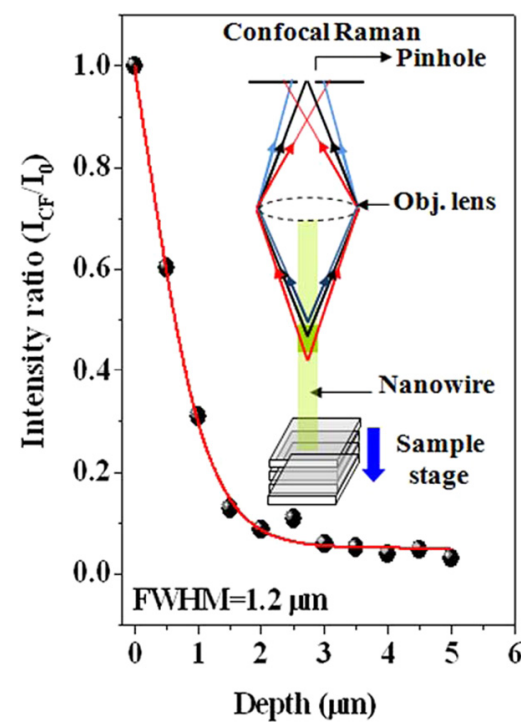

FIG. 2. The ratio of intensity of Raman scattered light through the pinhole $\left(I_{\mathrm{CF}}\right)$ and without the pinhole $\left(\mathrm{I}_{0}\right)$ as a function of depth from the focal plane from bulk InSb. Inset of the figure is the cartoon diagram explaining confocal measurements on NW (shown by the green bar). Only the black rays, from dark segment, pass the pinhole and reach the detector. The blue and red rays, from lighter segments of the NW, are blocked by the pinhole.

was recorded when the light was focused on the part of InSb segment near the interface with the InAs stem, while the topmost spectrum was acquired near the tip of the NW (refer to the side panel of Figure 3(a)). For standing [111] NWs, the
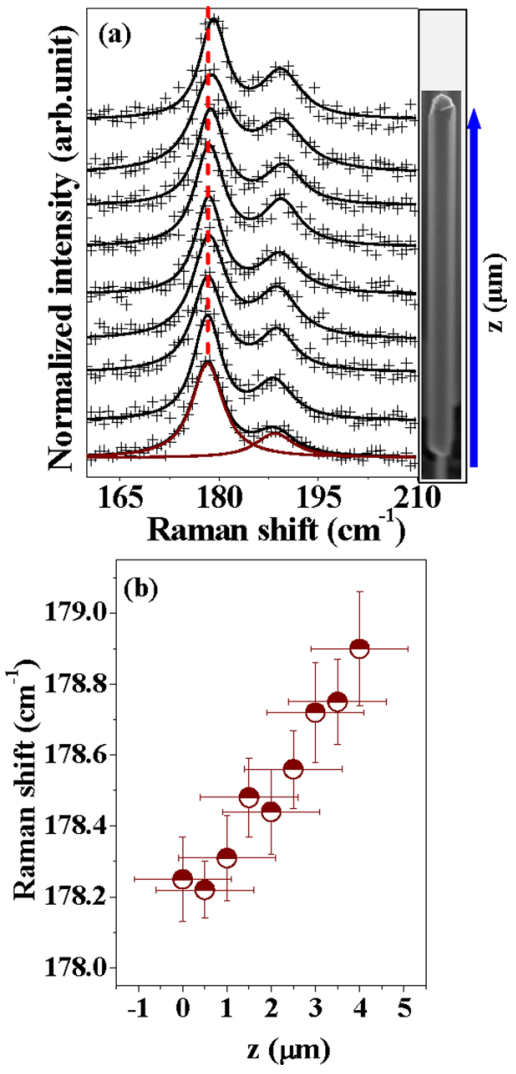

FIG. 3. (a) The evolution of Raman spectra along the axis of InAs/InSb NWs. The arrow marks the spectra, recorded from the bottom towards the tip of the NW. The vertical dashed line is drawn to show the shift in TO phonon frequency along the length of the NW. (b) The variation of the InSb TO phonon wavenumber along the length of the NW and horizontal error bar in each point indicates the step resolution. 
longitudinal optical (LO) phonon mode (at $\sim 191.0 \mathrm{~cm}^{-1}$ ) is not expected to appear in the back scattering geometry. ${ }^{16}$ The appearance of the LO phonon mode may be due to the breakdown of crystallinity of the lattice, as for bulk [111] InSb wafer we, indeed, did not observe this mode in the given experimental configuration. In this experimental configuration, the transverse optical (TO) phonon mode at $\sim 179.7 \mathrm{~cm}^{-1}$ is Raman active. ${ }^{17}$ Each spectrum in Figure 3(a) has been deconvoluted with two Lorentzian functions for TO and LO modes, keeping peak positions, widths and intensities as free fitting parameters. Deconvoluted spectra are shown as magenta lines for the lowest spectrum of Figure 3(a). The vertical red dashed line in Figure 3(a) marks the position of the TO peak of each curve to highlight its gradual shift in the spectra towards the tip of the NW. The Raman shift of the TO phonon mode along the length of the InSb NW, as obtained from the above curve fitting analysis, is shown in Figure 3(b). In the figure, we consider the bottom of the InSb segment near the interface with the InAs stem as the zero of the z-axis in Figure 3(b). Keeping in mind the depth resolution associated with our z-scan (shown in Figure $2)$, the horizontal error bar for each data point plotted in Figure 3(b) has been kept as $\pm 1.2 \mu \mathrm{m}$. We observe a monotonous change in Raman shift of the TO mode as the laser probe moves from the InAs/InSb interface towards the tip of the InSb segment (positive $\mathrm{z}$ as per our notation). The LO phonon mode also shifts towards the higher wavenumber as the TO phonon mode. However, the LO phonon mode is relatively weaker than the TO mode, and its peak position, as estimated from the curve fitting program, strongly depends on various fitting parameters. Thus, to discuss about the origin of Raman shift, we restrict ourselves only on the shift of the TO phonon mode.

Several possible explanations can be argued in order to interpret the observed TO phonon mode shifts. (i) As the InSb segments are grown on InAs stems, between InAs and $\mathrm{InSb}$ a transition region of $\operatorname{InAs}_{\mathrm{x}} \mathrm{Sb}_{1-\mathrm{x}}$ may exist. ${ }^{6}$ Thus, the observed shift in the phonon mode may be due to a small variable residual As concentration along the length of the NW. However, the formation of such alloy is, generally, confined within few $\mathrm{nm}$ near the heterointerface, while the length of our InSb segments is $\sim 4 \mu \mathrm{m}$. Moreover, InSb-like TO peak position in $\operatorname{InAs}_{\mathrm{x}} \mathrm{Sb}_{1-\mathrm{x}}$ does not change with As content. ${ }^{18}$ Thus, we rule out the possibility that the observed shift in the phonon mode in Figure 3(b) is due to a change in composition along the axis of the NW. (ii) The observed TO phonon shifts can be associated to phonon confinement effects. However, the diameter of the NWs, used in our study, is too large $(\sim 100-200 \mathrm{~nm})$ to shift their Raman peak. Hence, even a slight variation in the diameter of the NWs, from the base to tip, would not shift the phonon peak. (iii) To check whether misalignment in the optics is the origin of the shift in the TO mode, discussed above, the spectra were acquired with $\mathrm{z}$ scans in the opposite direction, i.e., when the laser was first focused at the tip of the NWs and the sample stage was moved up to access the other points along the axis towards the InAs stem. In this case, too, a similar shift in the TO phonon mode along the axis has been observed, as shown in Figure 3, excluding misalignment in the optics as possible reason for the shift. (iv) We rule out the possibility of laser heating and/or non-uniform heat dissipation along the axis of the NW to be the origin of the observed shift in TO phonon mode in Figure 3. In fact, we have recorded Stokes-anti-Stokes Raman spectra along the axis of the NWs [Figure S1 of the supplementary material $]^{14}$ and we did not observe any systematic change in the temperature: the average temperature remains $294 \pm 10 \mathrm{~K}$ [see the inset of Figure S1 in the supplementary material]. ${ }^{14}$ Furthermore, it is known that the separation between LO and TO phonon modes in InSb changes with temperature. ${ }^{19}$ From Figure 3(a), we find that both LO and TO phonon modes shift towards higher wavenumbers, nearly by same amount, i.e., approximately a constant separation between two modes is maintained in all spectra. This ensures that the local temperature difference is not the origin of the shift observed in the individual modes along the axis of the NW.

Another explanation could be associated to the presence of residual strain along the NWs. In fact, the phonon frequency of a crystal depends on the interatomic force constants. Any local distortion in a perfect crystal structure modifies the deformation potential-optical phonon interactions in semiconductors. ${ }^{20}$ This short range interaction is manifested in relative displacement of atoms at the zone center optical phonons. ${ }^{21}$ The strain tensor components, arising from the static distortion in the crystal, result in nonidentical movement of the atoms, and modify the phonon frequencies. The modified TO frequency can be related to the hydrostatic stress and shear stress along [111] ZB plane by $^{20} \Omega=\Omega_{0}+\Delta \Omega_{H}+(2 / 3) \Delta \Omega_{s}$, with $\Delta \Omega_{H}=\left(\sigma / 6 \Omega_{0}\right)$ $(p+2 q)\left(S_{11}+2 S_{12}\right)$ and $\Delta \Omega_{s}=\left(\sigma / 2 \Omega_{0}\right) r S_{44}$. Here, $\Omega_{0}$ is the phonon frequency of the unstrained crystal, $\sigma$ is the net stress in the crystal, $p, q$, and $r$ coordinates are related to deformation potential, and $S_{i j}$ are elastic compliance constants with respect to the cubic axis. The corresponding hydrostatic strain and shear strain in the crystal are $\varepsilon_{H}=\left(S_{11}+2 S_{12}\right)$ $(\sigma / 3)$ and $\varepsilon_{S}=\left(S_{44} / 2\right)(\sigma / 3)$, where $\varepsilon_{\mathrm{H}}$ and $\varepsilon_{\mathrm{S}}$ correspond to diagonal and off-diagonal components of the strain tensor matrix. Using Above equations along with the reported values of the physical constants $\left(S_{i j}, p, q, r\right)$ for InSb, ${ }^{20,22,23}$ and measured $\Delta \Omega=\Omega-\Omega_{0}$ from Figure $3(\mathrm{~b})$, the variation of hydrostatic and shear strain along the axis of InSb NW is plotted in Figure 4. The value of $\Omega_{0}$ has been taken as $179.7 \mathrm{~cm}^{-1}$. We find that both hydrostatic and shear strain do exist along the wire. They are maximum near the interface and gradually decrease along the axis of the NW. The measured shear strain varies from $0.11 \%$ to $0.06 \%$ and the hydrostatic strain varies from $0.28 \%$ to $0.14 \%$.

Next, we collected SAED patterns at the bottom, center, and top of the InSb segment in several NWs. Since electron diffraction has a poor resolution in determining the absolute value of the unit cell parameters (which could be related to the above measured weak strain), we focused the analysis on the determination of the distortion of cubic crystal structure, which relays on relative measurements on equivalent parameters.

At first, we evaluated deformation of the structure in [011] zone axis pattern by comparing the $d$ spacing along two equivalent (111) directions, one parallel and the other inclined to the NW axis (shown in Figure S2(a) of the 


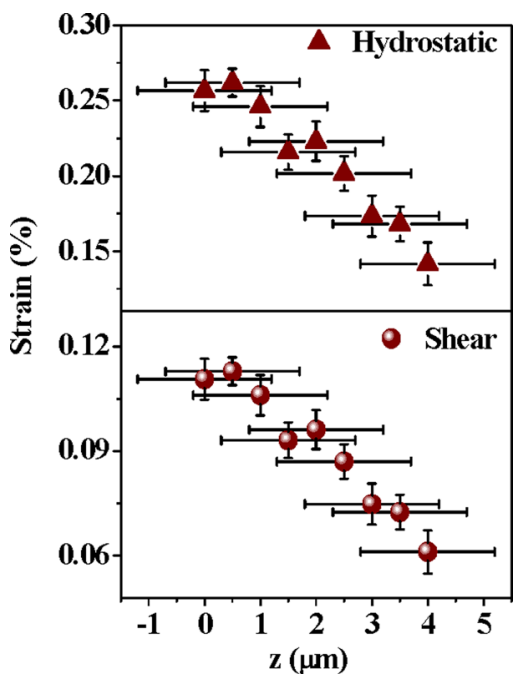

FIG. 4. Estimated variation of hydrostatic and shear strain along the axis of InAs/InSb NWs.

supplementary material). ${ }^{14}$ We derived the two equivalent $d_{111}$ spacing by measuring the distances between Friedel pair reflections of the third order (i.e., 333 and -3-3-3, see arrows Figure S2(a)). In all cases, the differences between the two $d$-spacing were within the experimental error while the maximum noticeable difference being $3.68 \pm 0.03 \AA$ against $3.72 \pm 0.03 \AA$.

Next, we measured the cell parameters on a 3-dimensional reconstruction of the reciprocal space (Figure S2(b) of the supplementary material ${ }^{14}$ obtained with the EDT technique. ${ }^{24}$ In EDT, a set of diffraction patterns are collected while the sample is rotated around the goniometer axis in steps of the order of $0.5^{\circ}$ for a total angular range of $60^{\circ}$. It is possible to obtain the unit cell parameters from the position of the diffracted spots in the three dimensional reconstruction, derived from the recorded patterns with the PETS software. ${ }^{25}$ To check possible distortions of the cubic structure, we indexed the cubic face centered cell with a primitive rhombohedral cell, which should have-in case of an undistorted lattice $-\mathrm{a}=\mathrm{b}=\mathrm{c}$ and $\alpha=\beta=\gamma=60^{\circ}$. In our NWs, the distortion in the lattice is found to be beyond the sensitivity of the technique. The maximum measured difference between the two lengths is $4.40 \pm 0.04 \AA$ against $4.44 \pm 0.04 \AA$ and between the two angles is $59.3 \pm 0.5^{\circ}$ against $59.9 \pm 0.5^{\circ}$.

In summary, confocal Raman measurements, which are highly sensitive to the atomic-scale local disorder in a crystal structure, allowed us to observe a weak residual strain of $\sim 0.2 \%$ in our NWs. The observed strain gradually decays from the interface towards their tip of the InAs/InSb NW. Conversely, the measured strain is extremely weak to be detected by SAED or EDT measurements, within the resolution limit of these experimental techniques. In fact, as most of the reports in the literature, claiming the strain relaxation at the interface in NWs, are based on electron diffraction techniques, the residual strain may not have been detected earlier. We believe that our studies may pave way for better understanding of strain release mechanisms in heterostructured NWs.

A.R. thanks the Abdus Salam International Center of Theoretical Physics (ICTP) for the TRIL fellowship to carry out the present collaborative research work.

${ }^{1}$ T. Ashley, A. B. Dean, C. T. Elliott, G. J. Pryce, A. D. Johnson, and H. Willis, Appl. Phys. Lett. 66, 481 (1995).

${ }^{2}$ K. A. Dick, Prog. Cryst. Growth Charact. Mater. 54, 138 (2008).

${ }^{3}$ H. A. Nilsson, P. Caroff, C. Thelander, M. Larsson, J. B. Wagner, L. Wernersson, L. Samuelson, and H. Q. Xu, Nano Lett. 9, 3151 (2009).

${ }^{4}$ M. T. Deng, C. L. Yu, G. Y. Hung, M. Larsson, P. Caroff, and H. Q. Xu, Nano Lett. 12, 6414 (2012).

${ }^{5}$ J. W. G. van den Berg, S. Nadj-Perge, V. S. Pribiag, S. R. Plissard, E. P. A. M. Bakkers, S. M. Frolov, and L. P. Kouwenhoven, Phys. Rev. Lett. 110, 066806 (2013).

${ }^{6}$ M. de la Mata, C. Magén, P. Caroff, and J. Arbiol, Nano Lett. 14, 6614 (2014).

${ }^{7}$ C. Y. Wen, M. C. Reuter, D. Su, E. A. Stach, and F. M. Ross, Nano Lett. 15, 1654 (2015).

${ }^{8}$ D. Ercolani, F. Rossi, Ang Li, S. Roddaro, V. Grillo, G. Salviati, F. Beltram, and L. Sorba, Nanotechnology 20, 505605 (2009).

${ }^{9}$ J. L. Taraci, M. J. Hytch, T. Clement, P. Peralta, M. R. McCartney, J. Drucker, and S. T. Picraux, Nanotechnology 16, 2365 (2005).

${ }^{10}$ M. W. Larsson, J. B. Wagner, M. Wallin, P. Håkansson, L. E. Fröberg, L. Samuelson, and L. R. Wallenberg, Nanotechnology 18, 015504 (2007).

${ }^{11}$ M. Montazeri, M. Fickenscher, L. M. Smith, H. E. Jackson, J. Y. Rice, J. H. Kang, Q. Gao, H. H. Tan, C. Jagadish, Y. Guo, J. Zou, M. E. Pistol, and C. E. Pryor, Nano Lett. 10, 880 (2010).

${ }^{12}$ S. Funk, A. Li, D. Ercolani, M. Gemmi, L. Sorba, and I. Zardo, ACS Nano 7, 1400 (2013).

${ }^{13}$ J. K. Panda, A. Roy, A. Singha, M. Gemmi, D. Ercolani, V. Pellegrini, and L. Sorba, Appl. Phys. Lett. 100, 143101 (2012).

${ }^{14}$ See supplementary material at http://dx.doi.org/10.1063/1.4929979 for the details.

${ }^{15}$ R. Tabaksblat, R. J. Meier, and B. J. Kip, Appl. Spectrosc. 46, 60 (1992).

${ }^{16}$ J. Wu, D. Zhang, Q. Lu, H. R. Gutierrez, and P. C. Eklund, Phys. Rev. B 81, 165415 (2010).

${ }^{17}$ K. Aoki, E. Anastassakis, and M. Cardona, Phys. Rev. B 30, 681 (1984).

${ }^{18}$ N. L. Rowell, D. J. Lockwood, G. Yu, Y. Z. Gao, X. Y. Gong, M. Aoyama, and T. Yamaguchi, J. Vac. Sci. Technol., A 22, 935 (2004).

${ }^{19}$ E. Liarokapis and E. Anastassakis, Phys. Rev. B 30, 2270 (1984).

${ }^{20} \mathrm{P}$. Y. Yu and M. Cardona, Fundamentals of Semiconductors, 3rd ed. (Springer, 2001), p. 122.

${ }^{21}$ F. Cerdeira, C. J. Buchenauer, F. H. Pollak, and M. Cardona, Phys. Rev. B 5, 580 (1972).

${ }^{22}$ R. M. Martin, Phys. Rev. B 1, 4005 (1970).

${ }^{23}$ M. I. Bell, Phys. Status Solidi B 53, 675 (1972).

${ }^{24}$ U. Kolb, T. Gorelik, C. Kübel, M. T. Otten, and D. Hubert, Ultramicroscopy 107, 507 (2007).

${ }^{25}$ L. Palatinus, PETS - software for processing of electron diffraction data, Institute of Physics, Praha, Czech Republic, 2011. 